\title{
Synthesis, characterization, and visible-light-driven photocatalytic performance of W-SBA15
}

\author{
Fei Chang ${ }^{\mathrm{a}, *}$, Jie Wang ${ }^{\mathrm{a}}$, Jieru Luo ${ }^{\mathrm{a}}$, Junrong Sun ${ }^{\mathrm{a}}$, Xuefeng $\mathrm{Hu}^{\mathrm{b}, *}$ \\ ${ }^{a}$ School of Environment and Architecture, University of Shanghai for Science and Technology, Shanghai 200093, PR China \\ ${ }^{\mathrm{b}}$ Key Laboratory of Coastal Zone Environmental Processes and Ecological Remediation, Yantai Institute of Coastal Zone Research, Chinese Academy of Sciences, Yantai, \\ Shandong 264003, PR China
}

G R A P H I C A L A B S T R A C T
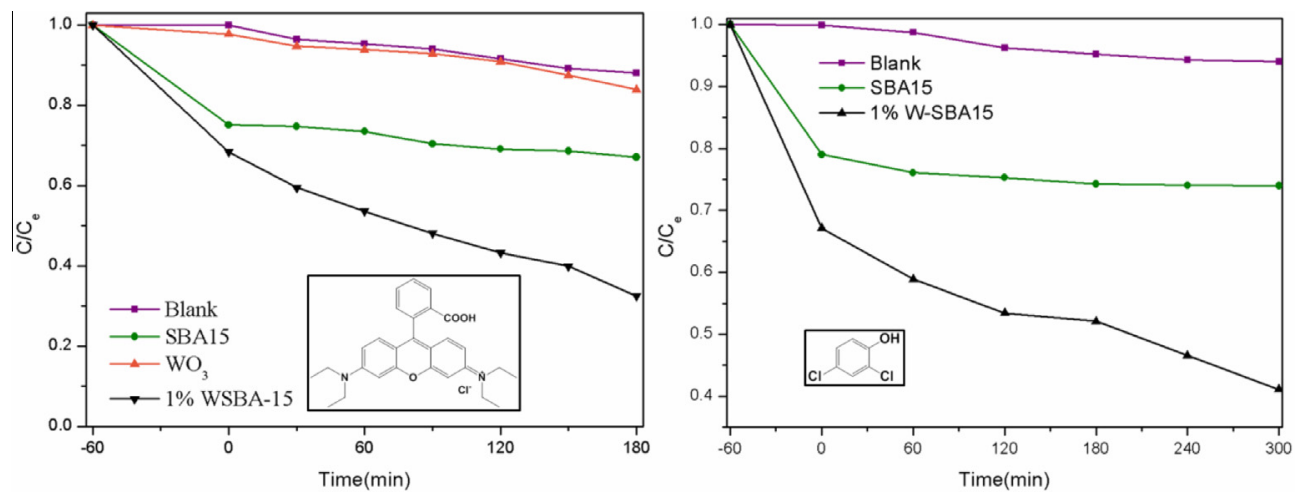

\section{A R T I C L E I N F O}

\section{Article history:}

Received 29 September 2015

Revised 29 January 2016

Accepted 30 January 2016

Available online 4 February 2016

\section{Keywords}

W-SBA15

Photocatalysis

Rhodamine B

2,4-Dichlorophenol

Mechanism

\begin{abstract}
A B S T R A C T
A series of tungsten-containing mesoporous SBA15 with different tungsten contents were prepared through a direct co-condensation sol-gel method and then analyzed by various techniques. XRD patterns revealed that the original mesoporous morphology was maintained after introducing tungsten species and forms of tungsten species were changed from incorporated tungsten to isolated tungsten species or low oligomeric tungsten oxide species, and finally to crystalline $\mathrm{WO}_{3}$ with the increase of tungsten precursor content, which were also proven by HRTEM images, UV-Vis DRS, and FT-IR spectra. The W-SBA15 samples exhibited satisfactory photocatalytic performance toward degradation of dye Rhodamine B (RhB) and 2,4-dichlorophenol (2,4-DCP). In particular, the best candidate, sample $10 \% \mathrm{~W}$ SBA15 showed an apparent reaction rate constant for RhB that was nearly 10 times as high as that of bulk $\mathrm{WO}_{3}$. The enhancement of photocatalytic capability was attributed to the mesoporous morphology with enlarged surface areas, negatively charged surface, and favorable tungsten forms such as incorporated tungsten, isolated tungsten or low oligomeric tungsten oxide species. In addition, active radicals trapping experiments and DMPO spin-trapping ESR spectra indicated that photogenerated holes were major oxidative species during photocatalysis.
\end{abstract}

(c) 2016 Elsevier Inc. All rights reserved.

\footnotetext{
* Corresponding authors.

E-mail addresses: feichang@usst.edu.cn (F. Chang),xfhu@yic.ac.cn (X. Hu).
} 


\section{Introduction}

Photocatalytic technology has attracted massive attention during last dozen years because of the powerful degradation capability for a wide range of undesirable chemical contaminants in water and air by using solar or artificial light irradiation [1-3]. Although $\mathrm{TiO}_{2}$ is deemed as a low cost and highly efficient photocatalyst with the unique chemical and thermal stability, practical applications of $\mathrm{TiO}_{2}$ are greatly restrained by the low quantum efficiency and irresponsivity to visible light [4,5]. In contrast, $\mathrm{WO}_{3}$ with a narrow band gap of $2.7 \mathrm{eV}$ can be activated in visible-light region. The major disadvantage of $\mathrm{WO}_{3}$ as a photocatalyst is the low photonic efficiency, as a result of the low conduction band level and the difficult of electrons consumption after activation [6]. In general, structural modification and morphology tailoring are both solutions to overcome the above-mentioned weaknesses [7-9].

The former method is relevant to the structural modification of $\mathrm{WO}_{3}$ by means of incorporating other semiconductors with appropriate electronic structures, metal and nonmetal elements, and doping with metal ions [9-18]. With suitable ingredients, accumulated electrons on conduction band of $\mathrm{WO}_{3}$ tend to be completely transferred and accordingly, the recombination rate of photogenerated hole-electron pairs is greatly depressed [15]. The latter approach is to modify synthetic routes to tailor morphology and size of nanostructured $\mathrm{WO}_{3}$ that provides not only abundant active sites for catalytic reactions but reduced diffusion distances for charge carriers to active sites [19-25]. To support or immobilize $\mathrm{WO}_{3}$ into an inert substrate is an efficient way to enhance photocatalytic behavior in terms of controlling the morphology and size of $\mathrm{WO}_{3}$. Mesoporous silicate SBA15 is generally selected as a support for photocatalytic applications because of the highly ordered two-dimensional hexagonal morphology, large specific surface area, adjustable pore size, good hydrothermal stability [26,27], and most importantly, the electron delocalization capacity by framework in comparison with any other porous silicate substrates [28-30].

Actually, $\mathrm{WO}_{3}$-containing SBA15 have been synthesized and employed to catalyze a variety of chemical transformations, such as oxidation of cyclopentene [31,32], desulfurization [33], dehydration [34], and metathesis [35,36]. The incorporation of tungsten source into SBA15 matrix induces the formation of different tungsten species forms, among which isolated tungsten species or low oligomeric tungsten oxide species with terminal $\mathrm{W}=0$ groups are deemed as actives sites to initiate above catalytic reactions [31-36]. Though these tungsten species are powerful to catalyze organic transformations under conventional conditions, as far as we know, there are no reports focusing on the photocatalytic evaluation of tungsten-containing SBA15 under visible-light irradiation. Therefore, in the present investigation, a series of W-containing SBA15 samples were prepared and characterized by a collection of analytical techniques. In this work, we showed that existed tungsten forms were transformed from highly dispersed tungsten species on SBA-15 to crystalline $\mathrm{WO}_{3}$ along with the increase of tungsten content. The as-prepared samples showed improved photocatalytic performance toward degradation of $\mathrm{RhB}$, an industrial dye, in comparison with bulk $\mathrm{WO}_{3}$, mainly owning to the mesoporous morphology with enlarged surface areas, relatively negative charged surface, and suitable tungsten species. In addition, these W-SBA15 samples were also quite efficient in catalytic degradation of 2,4-DCP, a colorless and non-biodegradable organic pollutant. However, from the aspect of potential application, the prepared W-SBA15 series needed further structural modification to obtain materials with excellent photocatalytic performance as well as good reusability.

\section{Experimental}

\subsection{Materials}

Pluronic P123 $\left(\mathrm{PEO}_{20} \mathrm{PPO}_{70} \mathrm{PEO}_{20}\right)$ was obtained from Aldrich Chemical Co. Sodium tungstate $\left(\mathrm{Na}_{2} \mathrm{WO}_{4} \cdot 2 \mathrm{H}_{2} \mathrm{O}\right)$ was purchased from ACROS Organics. Hydrochloric acid $(\mathrm{HCl})$, tetraethyl orthosilicate (TEOS), and other reagents were supplied by Sinopharm Chemical Reagent Co., Ltd. (Shanghai, China). All Chemicals were used directly for experiments without any further purification. Deionized water was used throughout the experiment.

\subsection{Preparation of $W$-SBA15 series}

In a typical synthesis, $\mathrm{P} 123$ (1.8 g) was dissolved in an $\mathrm{HCl}$ aqueous solution ( $2 \mathrm{M}, 58.5 \mathrm{~mL}$ ) and the mixture was stirred at $35^{\circ} \mathrm{C}$ for $6 \mathrm{~h}$ prior to the addition of TEOS (3.87 g). The mixture was vigorously stirred for $30 \mathrm{~min}$ before introducing a desired volume of $\mathrm{Na}_{2} \mathrm{WO}_{4} \cdot 2 \mathrm{H}_{2} \mathrm{O}$ aqueous solution $(0.2 \mathrm{M})$. After aging at $35{ }^{\circ} \mathrm{C}$ under moderate stirring for $24 \mathrm{~h}$, the resultant mixture was poured into a Teflon-lined stainless steel autoclave and kept at $100^{\circ} \mathrm{C}$ for $48 \mathrm{~h}$. Finally, a solid was collected, washed three times with distilled water and absolute ethanol, dried at $60^{\circ} \mathrm{C}$ overnight, and calcined at $550{ }^{\circ} \mathrm{C}$ for $5 \mathrm{~h}$ to give a target product, named as $x \mathrm{~W}$-SBA15, which $x$ stood for theoretical mass ratios of formed $\mathrm{WO}_{3} / \mathrm{SiO}_{2}$ (1\%, 5\%, $10 \%$ and $20 \%$ ). For comparison, pure SBA15 and $\mathrm{WO}_{3}$ were synthesized using a same procedure except that tungsten or silicon source was absent, respectively.

The color variation of all samples was shown in Fig. A1. As observed, pure SBA15 and $\mathrm{WO}_{3}$ were white and yellow in color, respectively. All as-prepared $\mathrm{W}$-SBA15 samples were white in color except sample $20 \% \mathrm{~W}$-SBA15 that possessed a pale yellow color, possibly owning to the formation of crystalline $\mathrm{WO}_{3}$ on surface.

\subsection{Characterization}

The X-ray diffraction (XRD) patterns were obtained with a Bruker D8 Advance $\mathrm{X}$-ray diffractometer using $\mathrm{Cu} \mathrm{K} \alpha$ radiation source ( $\lambda=1.5406 \AA$ ). UV-Vis diffuse reflectance spectra (UV-Vis DRS) were collected on a Shimadzu UV-2600 spectrophotometer with $\mathrm{BaSO}_{4}$ as a reference. Scanning electron microscopy (SEM) was performed on a Hitachi S-4800 field emission scanning electron microscopy. Transmission electron microscope (TEM), high resolution transmission electron microscopy (HRTEM), and selected area electron diffraction (SAED) patterns were recorded on a FEI Tecnai G2 F20 transmission electron microscope operated at $200 \mathrm{kV}$. HRTEM was analyzed by the Digital Micrograph software. X-ray photoelectron spectroscopy (XPS) measurements were performed by using an AMICUS System (Shimadzu) with Al Ka $(1486.8 \mathrm{eV})$ radiation. Binding energies were calibrated with the contaminated graphitic carbon adsorbed to the surface $(\mathrm{C} 1 \mathrm{~s}=284.6 \mathrm{~V})$. The specific surface areas were measured by nitrogen adsorption-desorption isotherms at $77 \mathrm{~K}$ using a Micromeritics TriStar II Plus apparatus. Before measurement, the samples were degassed at $423 \mathrm{~K}$ under vacuum for $6 \mathrm{~h}$. Surface charge was evaluated on a zeta potential analyzer (Malvern Zetasizer NANO ZS). The Fourier transform infrared spectra (FT-IR) were recorded with $\mathrm{KBr}$ pellet on a Thermo Nicolet Magna-IR 750 spectrometer.

\subsection{Photocatalytic performance}

Photocatalytic performance of all as-synthesized samples were evaluated by degrading RhB and 2,4-DCP under visible-light irradiation. A $300 \mathrm{~W}$ xenon lamp (CEL-HXF300, AuLight, Beijing) was 
utilized as a light source, equipping with a $420-780 \mathrm{~nm}$ cut-off filter. The distance between the light source and the surface of reaction suspension was $20 \mathrm{~cm}$. During measurement, the photocatalyst (20 mg) was dispersed in an aqueous solution of $\mathrm{RhB}\left(30 \mathrm{mg} \mathrm{L}^{-1}\right.$, $80 \mathrm{~mL}$ ) or 2,4-DCP (5 $\mathrm{mg} \mathrm{L}^{-1}, 80 \mathrm{~mL}$ ) and magnetically stirred for $1 \mathrm{~h}$ in dark to ensure an adsorption-desorption equilibrium before exposing to visible light irradiation. At every $30 \mathrm{~min}$ intervals, $4 \mathrm{~mL}$ aliquot was sampled, diluted, and centrifuged to remove suspended photocatalyst particles. The residual concentration of RhB and 2,4-DCP was analyzed by a UV-Vis spectrophotometer (Purkinje General T6) at the maximum absorption wavelength $554 \mathrm{~nm}$ and a UV-Vis spectrophotometer (Shimadzu UV-2600) at the maximum absorption wavelength $281 \mathrm{~nm}$, respectively.

To propose the photocatalytic mechanism, active radical species capturing experiments were accomplished. $5 \mathrm{mM}$ isopropanol (IPA) or disodium ethylenediaminetetraacetate (EDTA-2Na) was charged for trapping hydroxyl radicals $(\cdot \mathrm{OH})$ or produced holes $\left(\mathrm{h}^{+}\right.$) [37]. In addition, $25 \mu \mathrm{M}$ nitroblue tetrazolium (NBT) was added replacing $\mathrm{RhB}$ to detect superoxide radicals $\left({ }^{\circ} \mathrm{O}_{2}^{-}\right)$that might possibly generate during photocatalysis [38]. ESR signals of radicals spin-trapped by 5,5-dimethyl-1-pyrroline-N-oxide (DMPO) were measured on a FA200 spectrometer. The settings for spectrometer were as follows: $\lambda=425 \mathrm{~nm}$, center field, $323 \mathrm{mT}$; microwave frequency, $9.05 \mathrm{GHz}$; power, $0.998 \mathrm{~mW}$. DMPO spin-trapping ESR spectra of samples disperse in water for both DMPO-OH and DMPO- $\mathrm{O}_{2}^{-}$.

The reusability of sample $10 \% \mathrm{~W}-\mathrm{SBA} 15$ was estimated according to the following procedure: after first run, the catalyst powder was collected from aqueous solution by centrifugation, washed with deionized water and ethanol under ultrasonication for several times, dried overnight at $100^{\circ} \mathrm{C}$, and calcined at $550{ }^{\circ} \mathrm{C}$ for $2 \mathrm{~h}$. Afterwards, next recycling test was performed through the photocatalytic degradation of RhB using a same procedure described above.

\section{Results and discussion}

Low-angle XRD patterns of W-SBA15 series with different content of tungsten species are shown in Fig. 1A. All samples display three pronounced diffraction peaks corresponding to (100), (110), and (200) reflections of mesoporous silicon-based materials, indicating that the involvement of tungsten species into SBA15 didn't significantly affect the structure of $p 6 \mathrm{~mm}$ hexagonal symmetry [39]. The intensity of the (100) reflection decreases with the increase of tungsten species content, possibly owning to the change in electron density of tungsten incorporated materials, similar to XRD patterns of $\mathrm{WO}_{3}-\mathrm{MCM}-41$ [35]. In addition, a gradual shift of the (100) diffraction peak toward lower angle with improving amounts of tungsten species is observed, revealing the large cell diameter as a result of the replacement of $\mathrm{Si}-\mathrm{O}$ band by relatively long $\mathrm{W}-\mathrm{O}$ band [36]. A broad diffraction peak at nearly $25^{\circ}$ indicates the existence of amorphous or microcrystalline silica, as depicted in Fig. 1B. No characteristic diffraction peaks of $\mathrm{WO}_{3}$ is found, even the content of tungsten species reached $5 \%$, revealing that tungsten species were incorporated into the framework or evenly dispersed on surface of SBA15, in accordance with the result of Low-angle XRD patterns. The sample $10 \% \mathrm{~W}-\mathrm{SBA}-15$ displayed feeble peaks at $23.2^{\circ}, 23.6^{\circ}, 24.4^{\circ}, 33.3^{\circ}$, characteristic of microcrystalline $\mathrm{WO}_{3}$ formed [34]. A continuous increase of tungsten species content up to $20 \%$ leads to XRD patterns with evident and sharp peaks, owning to the phase segregation toward the formation of crystalline $\mathrm{WO}_{3}$ [40].

UV-Vis diffuse reflectance spectra of obtained W-SBA15 samples, SBA-15, bare $\mathrm{WO}_{3}$, and $\mathrm{Na}_{2} \mathrm{WO}_{4}$ were measured and displayed in Fig. 2A. It is well known that this technique is reliable to differentiate incorporated metal species from extra-framework metal oxides in different mesostructures [41]. Obviously, there are no evident bands in the spectrum of SBA-15. A distinct band around $230 \mathrm{~nm}$ in $\mathrm{Na}_{2} \mathrm{WO}_{4}$ corresponds to the spinel structure and isolated $\left[\mathrm{WO}_{4}\right]^{2-}$ tetrahedrons $[31,42]$. All W-SBA15 samples have the band mentioned above, which indicates the presence of highly dispersed tungsten species incorporated in silica framework. An absorption band centered at $290 \mathrm{~nm}$ in samples from $5 \% \mathrm{~W}-\mathrm{SBA} 15$ to $20 \% \mathrm{~W}$-SBA15 is assigned to isolated tungsten species or low oligomeric tungsten oxide species. In addition, a broad band around $400 \mathrm{~nm}$ that is weak in sample $10 \% \mathrm{~W}$-SBA15 and quite prominent in sample $20 \% \mathrm{~W}-\mathrm{SBA} 15$, can be ascribed to the extra-framework $\mathrm{WO}_{3}$ crystals or clusters by comparison with the spectrum of bare $\mathrm{WO}_{3}[31,42]$. As a result, with the increase of tungsten source content, tungsten species were able to transform from incorporated tungsten species in silica framework to $\mathrm{WO}_{3}$ crystalline or clusters on the surface of SBA-15, in agreement with the color variation of samples and wide-angle XRD patterns.

The $\mathrm{N}_{2}$ adsorption-desorption isotherms are shown in Fig. 2B. Basing upon IUPAC classification, all isotherms are of type IV with a H1 hysteresis loop that is a typical adsorption for mesoporous samples with one-dimensional cylindrical channels [34,43]. In addition, a sharp inflection in these isotherms occurs at relative pressure of 0.6-0.8, demonstrating the filling of the uniform mesopores due to the capillary condensation of mesoporous materials [36]. The position where the inflection begins is relevant to pore sizes and the sharpness of these inflections indicates the
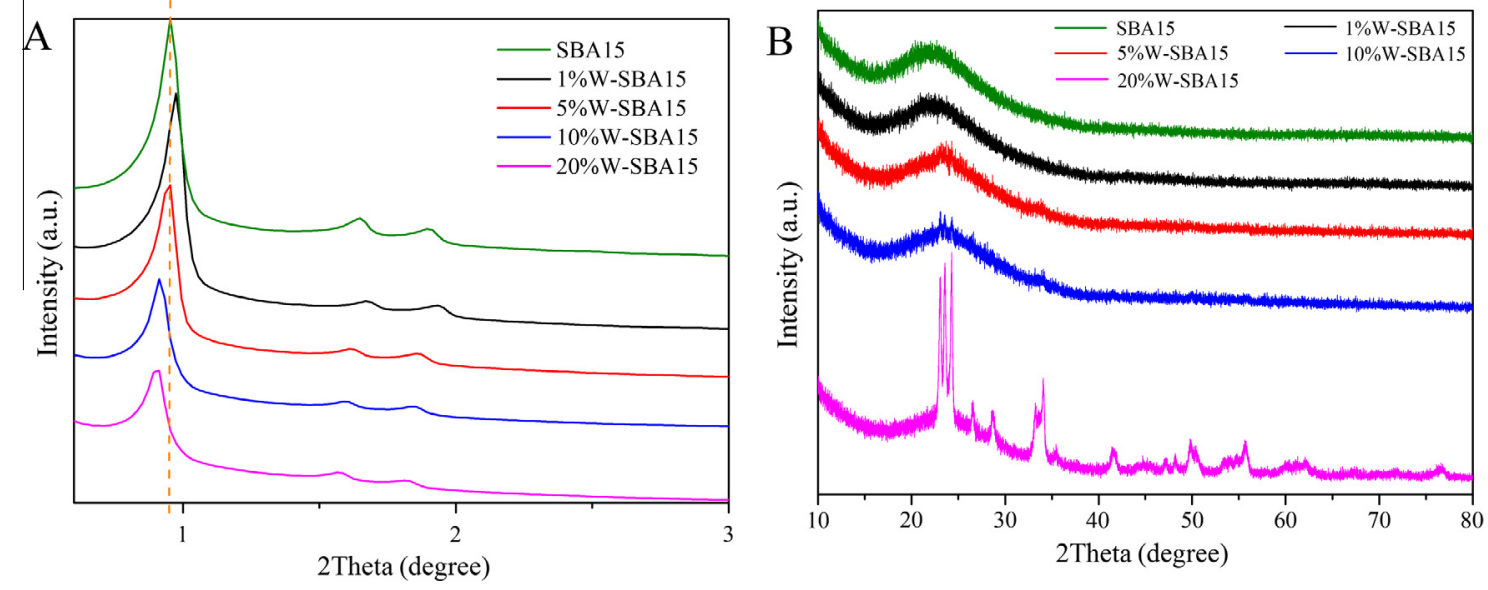

Fig. 1. Low-angle (A) and wide-angle (B) XRD patterns of pure SBA-15 and W-SBA15 series. 

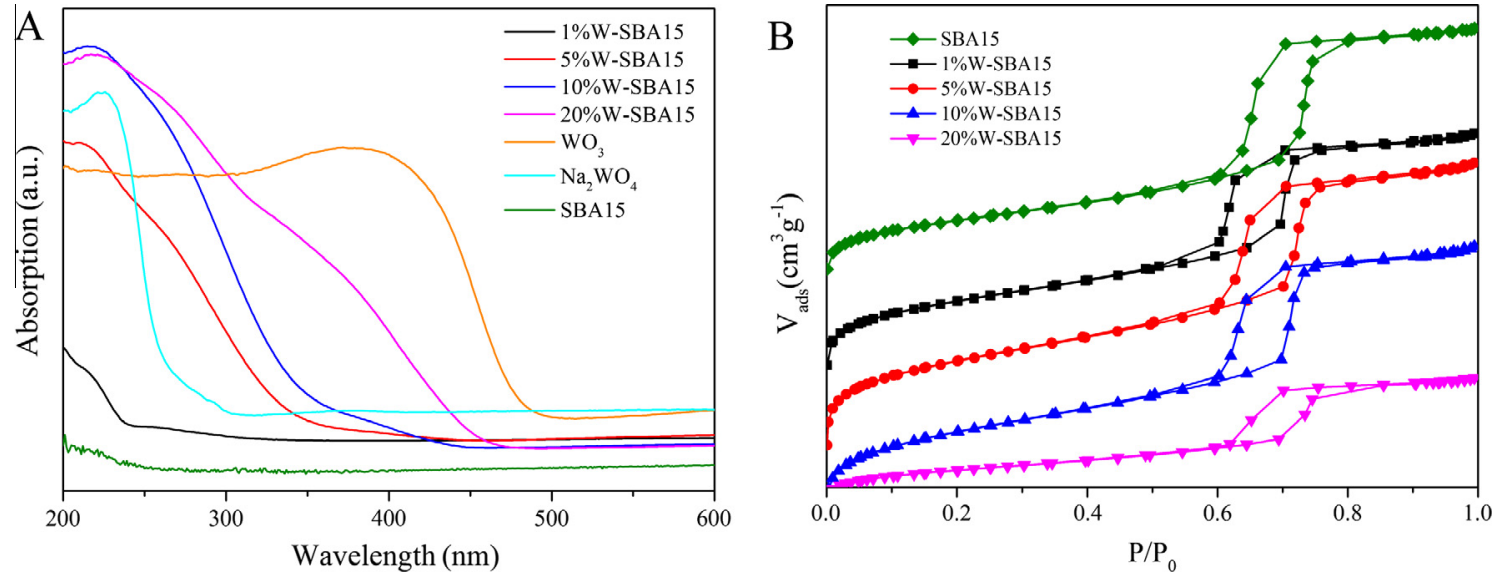

Fig. 2. UV-Vis DRS spectra of pure SBA-15, W-SBA15 series, bare $\mathrm{WO}_{3}$ and $\mathrm{Na}_{2} \mathrm{WO}_{4}(\mathrm{~A}), \mathrm{N}_{2}$ adsorption-desorption isotherms of pure $\mathrm{SBA}-15$ and $\mathrm{W}-\mathrm{SBA} 15$ series (B).

uniformity of the mesopore size distribution [36]. The BET surface area, pore volume, and pore diameter of all samples are listed in Table 1. It is evident that W-SBA15 series possess relatively large specific surface areas in comparison with bare SBA15, possibly attributed to the disrupted periodic pore structures by the introduction of tungsten heteroatoms, similar to the variation of $S_{B E T}$ of TiSBA15 series [44]. In addition, the increase of specific surface area with W contents may also be induced by the presence of microcrystalline $\mathrm{WO}_{3}$ that produces certain rugosity on surface of SBA15 when $\mathrm{W}$ content is below $20 \%$. Otherwise, the $S_{B E T}$ value of sample $20 \% \mathrm{~W}$ SBA15 is dramatically reduced, which may be induced by the formation of large $\mathrm{WO}_{3}$ crystals among $\mathrm{SiO}_{2}$ particles (TEM images), or the occupation of accumulated crystals $\mathrm{WO}_{3}$ into channels and onto surface of SBA15 [43,45], which is in agreement with the variation of pore diameter, as seen in Table 1 and Fig. A2.

SEM images of all tungsten-containing SBA15 samples are displayed in Fig. A3, revealing the maintenance of original welldefined wheat-like macrostructures of pure SBA15. These particles aggregate to form uniform rope-like morphology. Fig. 3A and $B$ exhibit TEM images of sample $10 \% \mathrm{~W}$-SBA15 and $20 \% \mathrm{~W}$-SBA15. As can be seen, some black spots on surface of SBA15, as circled by yellow lines are crystalline $\mathrm{WO}_{3}$ particles, which are further confirmed by the presence of elements W, O, and Si from EDX analyses of sample $10 \% \mathrm{~W}-\mathrm{SBA} 15$ and $20 \% \mathrm{~W}-\mathrm{SBA} 15$ in Fig. $3 \mathrm{C}$ and D. In addition, a well-ordered hexagonal array of mesoporous sample $10 \% \mathrm{~W}-\mathrm{SBA} 15$ is observed in Fig. 3E, indicating that the introduction of tungsten species did not obviously destruct the original structure of SBA15. The HRTEM image of 20\%W-SBA15 in Fig. 3F clarifies the highly crystalline nature and evident lattice fringes. These well-resolved lattice fringes with interplanar distance of $0.365 \mathrm{~nm}$ correspond to (200) crystallographic planes of $\mathrm{WO}_{3}$, further confirming the formation of crystalline $\mathrm{WO}_{3}$ on surface of SBA15. In addition, the SAED pattern in the inset of Fig. 3F exhibits periodical diffraction spots arrays indicative of single-crystalline characteristic of $\mathrm{WO}_{3}$, which is consistent with XRD patterns and UV-Vis DRS spectra.

Table 1

Physicochemical property of SBA15 samples.

\begin{tabular}{llll}
\hline Sample & $\begin{array}{l}\text { Surface area } \\
\left(\mathrm{m}^{2} / \mathrm{g}\right)\end{array}$ & $\begin{array}{l}\text { Pore volume } \\
\left(\mathrm{cm}^{3} / \mathrm{g}\right)\end{array}$ & $\begin{array}{l}\text { Pore diameter } \\
(\mathrm{nm})\end{array}$ \\
\hline SBA15 & 554 & 0.828 & 7.70 \\
1\%W-SBA15 & 677 & 0.731 & 7.00 \\
5\%W-SBA15 & 805 & 0.842 & 7.37 \\
10\%W-SBA15 & 783 & 0.792 & 7.18 \\
20\%W-SBA15 & 310 & 0.393 & 7.68 \\
\hline
\end{tabular}

XPS analysis of sample $10 \% \mathrm{~W}-\mathrm{SBA} 15$ was conducted to measure the chemical composition and surface valence states and is shown in Fig. 4. Peak positions in spectra were calibrated with $\mathrm{C} 1 \mathrm{~s}$ at $284.6 \mathrm{eV}$. Full scan XPS spectrum in Fig. 4A demonstrates the presence of elements $\mathrm{Si}, \mathrm{O}$, and $\mathrm{W}$, as expected. The XPS spectrum of $\mathrm{W}$ 4f can be deconvoluted to four peaks, as shown in Fig. 4B. Two peaks at $35.3 \mathrm{eV}$ and $37.45 \mathrm{eV}$ are characteristics of $\mathrm{W}^{5+}$ species, and another two at $36 \mathrm{eV}$ and $38.15 \mathrm{eV}$ are attributed to $\mathrm{W}^{6+}$ species [46]. The peak centered at $103.6 \mathrm{eV}$ in XPS spectrum of Si $2 \mathrm{p}$ is relevant to the presence of $\mathrm{Si}^{4+}$ in SBA15 framework [46] and a peak at $532.8 \mathrm{eV}$ in XPS spectrum of $\mathrm{O} 1 \mathrm{~s}$ is assigned to the $\mathrm{O}$ in $\mathrm{Si}-\mathrm{O}-\mathrm{Si}$ environment [47], as shown in Fig. A4.

FT-IR spectra in Fig. 5 depict a comparison of W-SBA15 series in chemical structure. As shown in Fig. 5, a broad and strong absorption peak centered at $3427 \mathrm{~cm}^{-1}$ is indexed to the stretching vibration mode of hydrogen-bonded $\mathrm{Si}-\mathrm{OH}$ moieties [43]. Bands around $1089 \mathrm{~cm}^{-1}$ is assigned to asymmetric stretching vibration of $\mathrm{Si}-\mathrm{O}-\mathrm{Si}$ bond while symmetric stretching and bending vibrations give rise to bands at nearly 803 and 467 [34,42]. It is realized that the peak at $963 \mathrm{~cm}^{-1}$ is a characteristic stretching $\mathrm{Si}-\mathrm{O}$ vibration mode perturbed by neighboring metal ions when the incorporation of metal ions in silica framework occurs [32,34]. Therefore, the existence of absorption peak at $963 \mathrm{~cm}^{-1}$ can directly prove the isomorphous substitution of Si by W ions in framework. However, the intensity of this peak slightly decreases from sample 5\%W-SBA15 to $20 \% \mathrm{~W}-\mathrm{SBA}-15$, possibly owing to the presence of extraframework crystalline $\mathrm{WO}_{3}$ [46], which is in agreement with the results from XRD patterns, UV-Vis DRS spectra, and TEM images.

The photocatalytic performance of obtained samples was measured through degradation of dye RhB and 2,4-DCP under visiblelight irradiation. As represented in Fig. $6 \mathrm{~A}$, similar to the outcome of direct photolysis of $\mathrm{RhB}$, bare $\mathrm{WO}_{3}$ shows a quite low photocatalytic performance toward degradation of RhB. The low photocatalytic behavior was resulted from the high recombination rate of charge carriers that was caused by the low conduction band level of bare $\mathrm{WO}_{3}$ [7-9]. Pure SBA-15, with mesoporous structure and a large specific surface area, can adsorb $24.9 \% \mathrm{RhB}$ in dark and exert no significant catalytic effect afterwards because of the lack of reactive sites. In contrast, sample $1 \% \mathrm{~W}-\mathrm{SBA} 15$ provides an enhanced adsorption ability comparing with SBA-15 and photocatalytic degradation efficiency close to $70 \%$ under identical conditions, mainly ascribing to the following factors. First, all W-SBA15 samples, except 20\%W-SBA-15, have much larger specific surface areas and more rough surface than pure SBA-15, ensuring the sufficient area with abundant active sites to interact with, subsequently adsorb, and finally destruct RhB molecules. Second, zeta potential values of both $10 \% \mathrm{~W}-\mathrm{SBA} 15$ and pure SBA15 


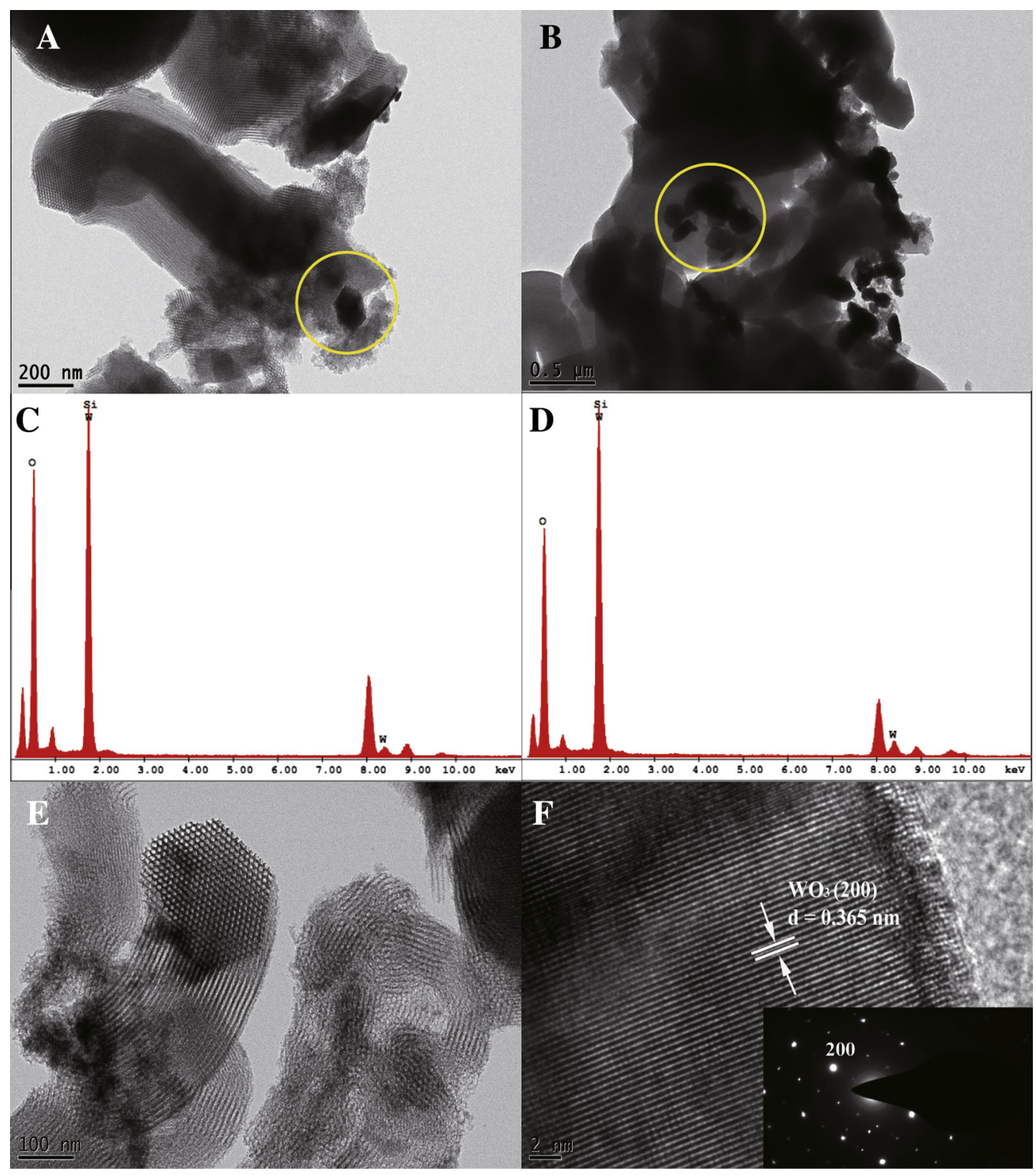

Fig. 3. TEM images of samples 10\%W-SBA15 (A) and 20\%W-SBA15 (B); EDX patterns of samples 10\%W-SBA15 (C) and 20\%W-SBA15 (D) from circled areas above; TEM image of $10 \% \mathrm{~W}-\mathrm{SBA} 15$ (E); HRTEM image and SAED pattern (inset) of 20\%W-SBA15 (F).
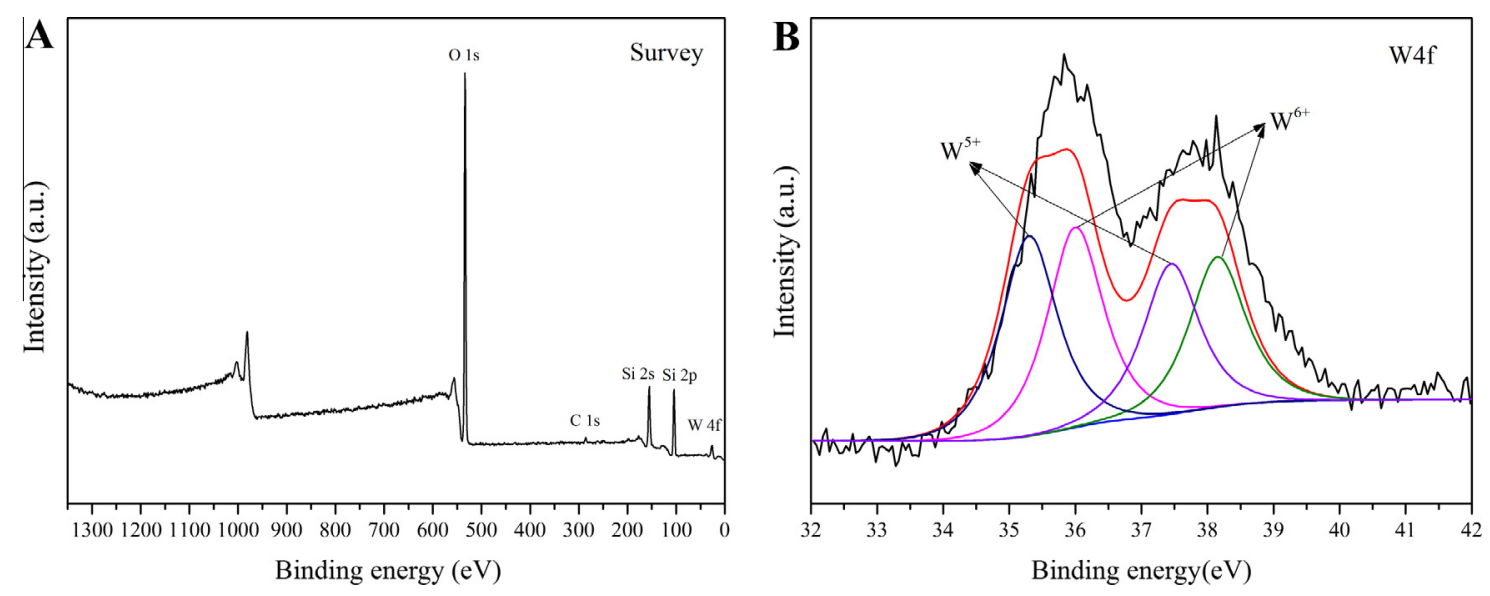

Fig. 4. XPS spectra of sample 10\%W-SBA15: survey spectrum (A), high-resolution spectrum of $\mathrm{W} 4 \mathrm{f}(\mathrm{B})$. 


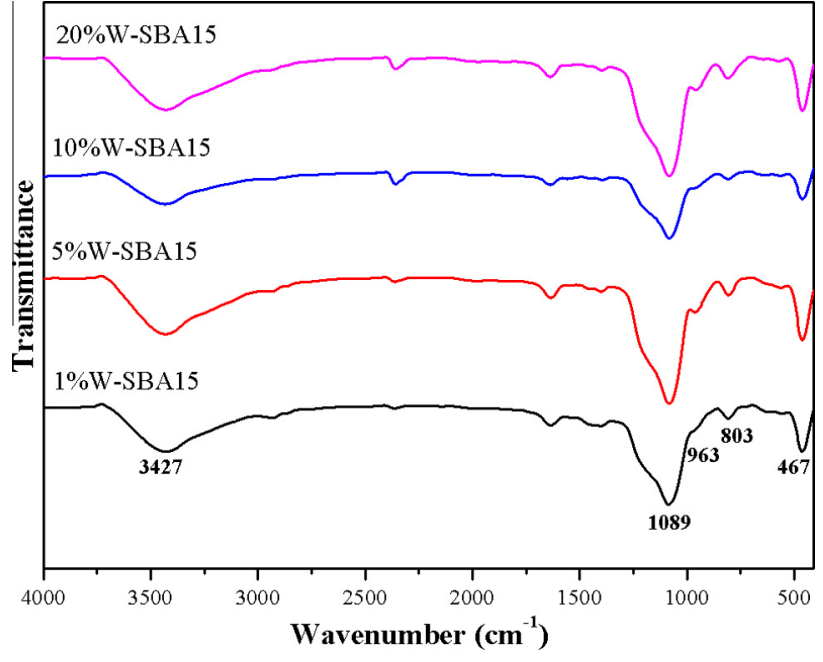

Fig. 5. FT-IR spectra of as-synthesized W-SBA15 series.

samples were determined as $-27.6 \mathrm{mV}$ and $-18.2 \mathrm{mV}$, revealing the more negatively charged surface of W-SBA15 than that of pure SBA15. Accordingly, more negative charges on surface favor interacting with and adsorbing cationic RhB molecules [48], thus benefiting the photocatalytic process. Photocatalytic capability comparison of W-SBA15 series is displayed in Fig. 6B. Obviously, photocatalytic performance is enhanced with the increase of the tungsten species content and approaches to a maximum value as the mass ratio of $\mathrm{WO}_{3} / \mathrm{SiO}_{2}$ is fixed at $10 \%$. Beyond that, the photo- catalytic performance decreases. The corresponding apparent reaction rate constants were calculated through a pseudo-first-order model, shown in Fig. 6C. Obviously, sample 10\%W-SBA-15 presents the largest apparent reaction rate constant that is nearly 10 times as high as bare $\mathrm{WO}_{3}$. The variation of photocatalytic capability can be mainly ascribed to both the content and nature of tungsten species involved. On the one hand, the increase of tungsten species content tends to supply abundant reactive sites and thus improving the photocatalytic behavior. On the other hand, the excessive addition of tungsten source easily promotes the form transformation from incorporated tungsten species to isolated tungsten species or low oligomeric tungsten oxide species, and finally to crystalline $\mathrm{WO}_{3}$. It has been reported that incorporated tungsten species, isolated tungsten species, and low oligomeric tungsten oxide species, rather than crystalline $\mathrm{WO}_{3}$ were powerful active sites for a variety of reactions $[31,32,46,49]$. In our study, three above tungsten species forms were indeed beneficial to the enhancement of photocatalytic efficiency, possibly owing to the quite intimate connection between framework of SBA15 and tungsten species, thus facilitating to transfer photogenerated electrons to SBA15 matrix and further transfer them by means of electron delocalization capacity from SBA15 framework [30], effectively avoiding the recombination of charge carriers. However, crystalline $\mathrm{WO}_{3}$ was of almost no photocatalytic ability, resulting from the relatively positive conduction band position and thereby high recombination of electron-hole pairs [6]. As a result, the enhancement of photocatalytic performance over W-SBA15 series are mainly attributed to the mesoporous morphology with enlarged surface areas, the delocalization capacity of silicate framework to efficiently transfer electrons, relatively negative charged surface, and
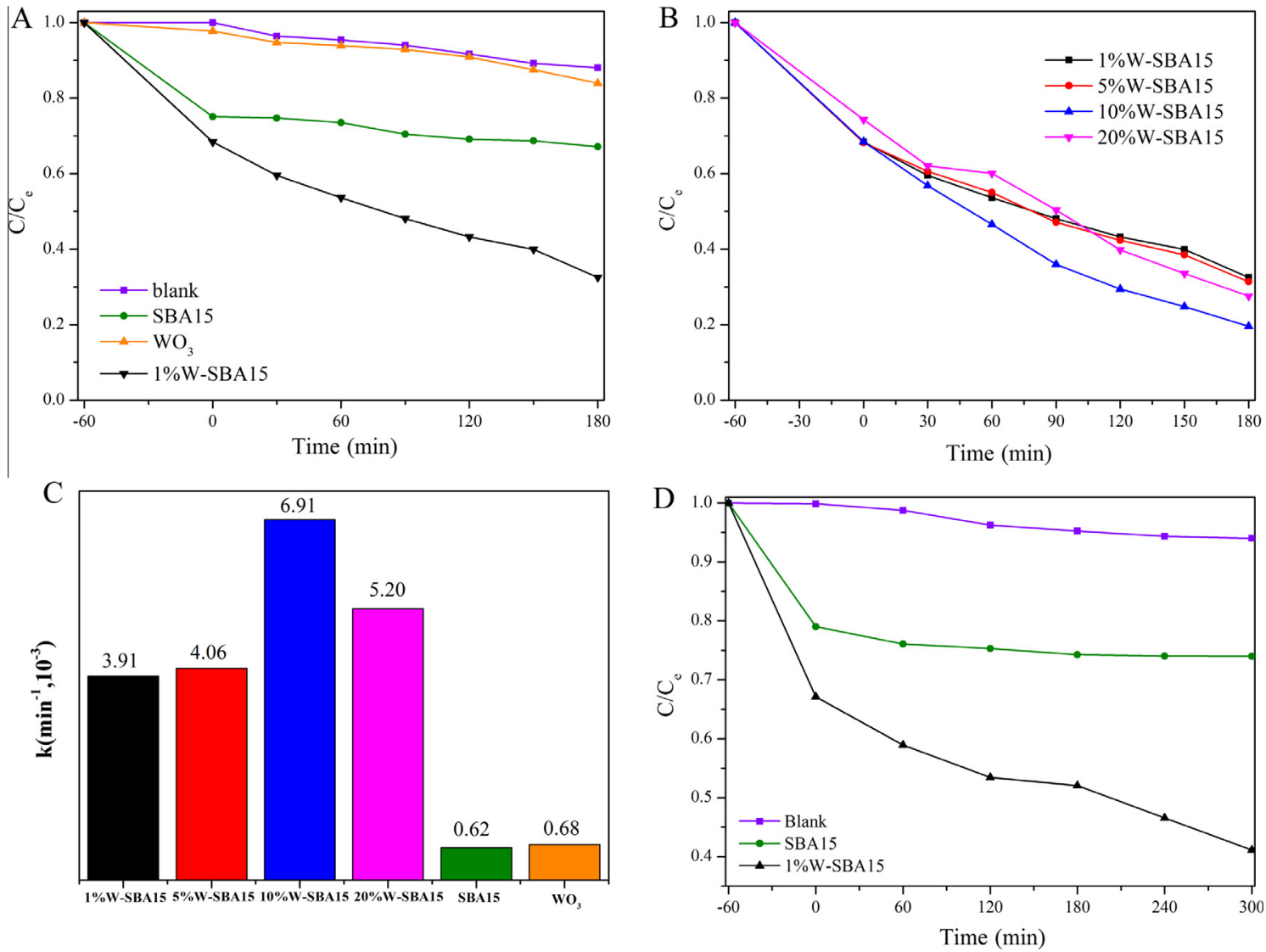

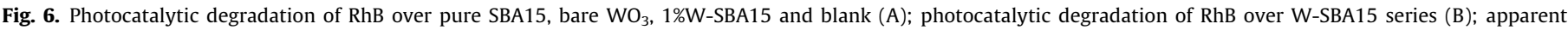
reaction rate constants over various catalysts for degradation of RhB (C); photocatalytic degradation of 2,4-DCP over pure SBA15, 1\%W-SBA15, and blank (D). 

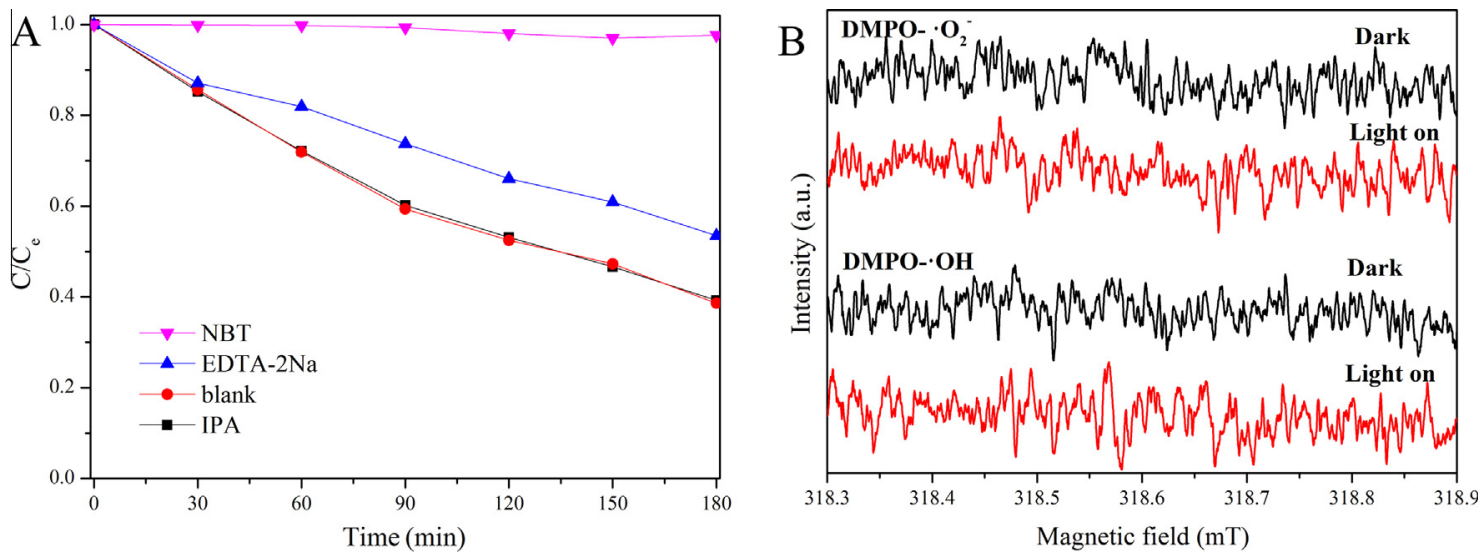

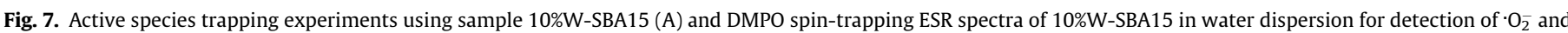
OH radicals.
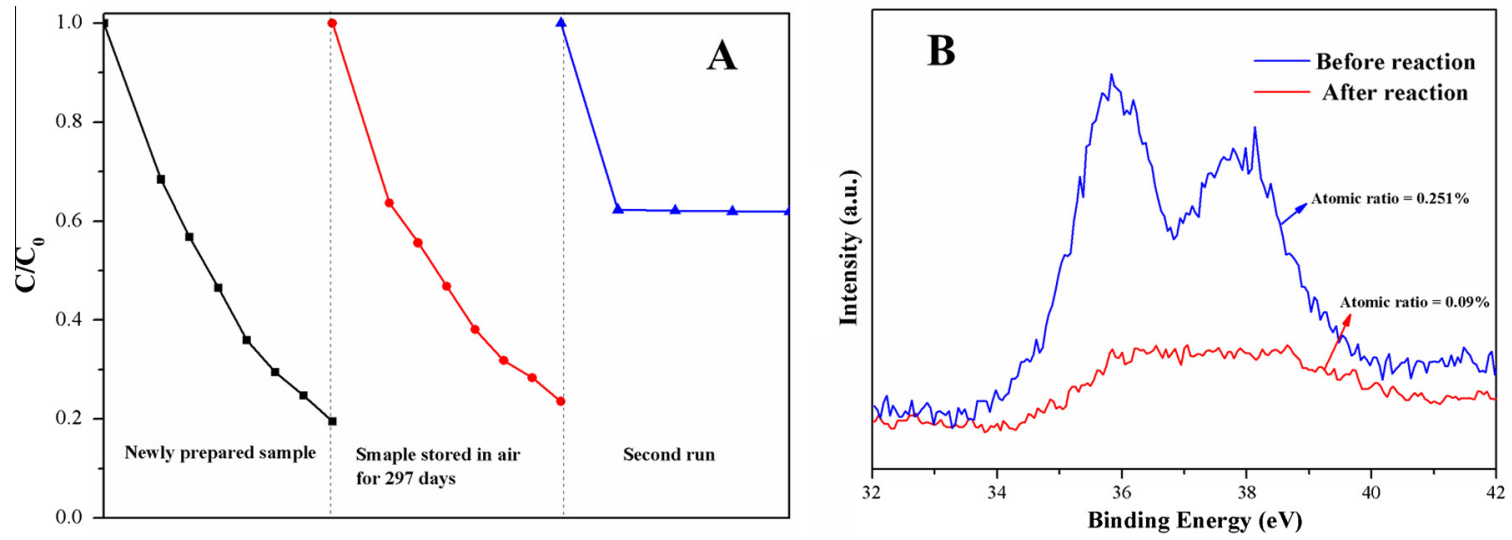

Fig. 8. The stability and reusability of sample 10\%W-SBA-15 (A) for photocatalytic performance; XPS spectra measured before and after photocatalytic reactions (B).

suitable tungsten forms. In order to rule out the possibility of dyesensitized path, a colorless organic pollutant 2,4-DCP, was typically chosen to evaluate the visible-light-driven photocatalytic performance of sample 1\%W-SBA15, as depicted in Fig. 6D. As can be seen, an obvious 2,4-DCP degradation up to $60 \%$ is observed after a photocatalytic treatment for $5 \mathrm{~h}$.

To propose a photocatalytic mechanism, main active radical species during photocatalytic reactions were detected. It is noted that in Fig. 7A, $X$-axis stands for the residue concentration of RhB and NBT when IPA or EDTA-2Na, and NBT were added to entrap $\cdot \mathrm{OH}$ or holes, and $\mathrm{O}_{2}^{-}$radicals, respectively. As shown in Fig. 7A, there is almost no change in the degradation of RhB after involvement of IPA, indicating that $\mathrm{OH}$ radicals are not the dominant oxidative species. In addition, the transformation percentage of NBT can be omitted, revealing the absence of ${ }^{\circ} \mathrm{O}_{2}^{-}$radicals [38]. In contrast, the addition of EDTA-2Na can greatly restrain the photocatalytic performance, confirming that holes are major reactive species, instead of both $\mathrm{OH}$ and ${ }^{-} \mathrm{O}_{2}^{-}$radicals. The conclusion was also supported by DMPO spin-trapping ESR spectra in Fig. 7B. Neither signals of DMPO- ${ }^{-} \mathrm{O}_{2}^{-}$or DMPO- $\mathrm{OH}$ adducts are found, further proving the absence of the two oxidative radicals that generally play an important role in photocatalytic processes. Therefore, the degradation of organic compounds in this study was mainly triggered by residue holes on valence band, and simultaneously photogenerated electrons were efficiently transferred through SBA-15 framework. So the recombination of hole-electron pairs was greatly depressed and correspondingly, the photocatalytic performance was significantly enhanced.
The stability and reusability of sample $10 \% \mathrm{~W}-\mathrm{SBA}-15$ were evaluated through a same procedure taken as above in Fig. 8A. It is obvious that this sample was robust and exerted a high photocatalytic efficiency even stored in air for 297 days. However, the photocatalytic efficiency of $10 \% \mathrm{~W}-\mathrm{SBA}-15$ after first recycling was quite low. To account for the above-mentioned phenomenon, XPS analysis of the sample before and after photocatalysis was thus tested and shown in Fig. 8B. Clearly, atomic ratio of tungsten was significantly reduced from $0.25 \%$ (before reaction) to $0.09 \%$ (after reaction), revealing that some tungsten species or clusters, especially crystalline $\mathrm{WO}_{3}$ were leached from surface of SBA15, and thus induced the loss of active sites required for photocatalytic processes. In our opinion, the leaching of tungsten species from SBA15 matrix was possibly attributed to free-radical reactions during special catalytic processes triggered by photoirradiation, since it was rarely taken place in conventional catalytic reactions $[31,42,46]$. As a result, further work should be undertaken and focused on both the investigation of structural variation in detail after photocatalytic reactions, and structural modifications to achieve W-SBA15 derivatives with satisfactory photocatalytic performance, stability, and reusability upon visible-light irradiation for the sake of real applications in environmental remediation and protection.

\section{Conclusion}

In this investigation, tungsten-containing mesoporous SBA15 materials were fabricated by means of a direct co-condensation 
sol-gel route and characterized by a collection of analytical techniques. Original mesoporous morphology was maintained after introducing tungsten resource and the tungsten form was changed from incorporated tungsten species to isolated tungsten species or low oligomeric tungsten oxide species, and finally to crystalline $\mathrm{WO}_{3}$ as long as the tungsten precursor content was increased. These W-SBA15 samples exhibited significantly high photocatalytic ability for RhB decomposition in comparison with bulk $\mathrm{WO}_{3}$. Especially, sample $10 \% \mathrm{~W}$-SBA15 showed the largest apparent reaction rate constant among W-SBA15 series that is nearly 10 times as high as bulk $\mathrm{WO}_{3}$. In addition, these samples were also efficient in photocatalytic degradation of 2,4-DCP. The enhancement of photocatalytic behavior is owed to the enlarged surface areas, relatively negative charged surface, and suitable tungsten forms. Active species trapping experiments confirmed that photoinduced holes instead of ${ }^{\circ} \mathrm{O}_{2}^{-}$and ${ }^{\circ} \mathrm{OH}$ radicals exerted major roles during photocatalytic degradation reactions, which was also proven by the DMPO spin-trapping ESR spectra. Eventually, further structural modification of W-SBA15 series was necessary to obtain materials with excellent photocatalytic performance as well as good reusability.

\section{Acknowledgements}

We are grateful to the National Natural Science Foundation of China (Grant number 21207089), the project-sponsored by SRF for ROCS, SEM., and the Hujiang Foundation of China (B14003) for financial support.

\section{Appendix A. Supplementary material}

Supplementary data associated with this article can be found, in the online version, at http://dx.doi.org/10.1016/j.jcis.2016.01.077.

\section{References}

[1] C. Chen, W. Ma, J. Zhao, Chem. Soc. Rev. 39 (2010) 4206-4219.

[2] A. Kubacka, M. Fernandez-Garcia, G. Colon, Chem. Rev. 112 (2012) 1555-1614.

[3] A. Kudo, Y. Miseki, Chem. Soc. Rev. 38 (2009) 253-278.

[4] X. Chen, S.S. Mao, Chem. Rev. 107 (2007) 2891-2959.

[5] I.K. Konstantinou, T.A. Albanis, Appl. Catal. B 49 (2004) 1-14.

[6] A. Hiskia, A. Mylonas, E. Chem, Soc. Rev. 30 (2001) 62-69.

[7] A.J.E. Rettie, K.C. Klavetter, J.F. Lin, A. Dolocan, H. Celio, A. Ishiekwene, H.L. Bolton, K.N. Pearson, N.T. Hahn, C.B. Mullins, Chem. Mater. 26 (2014) 16701677.

[8] A. Yan, C. Xie, F. Huang, S. Zhang, S. Zhang, J. Alloy Compd. 610 (2014) 132-137.

[9] M.S. Bazarjani, M. Hojamberdiev, K. Morita, G. Zhu, G. Cherkashinin, C. Fasel, T. Herrmann, H. Breitzke, A. Gurlo, R. Riedel, J. Am. Chem. Soc. 135 (2013) $4467-$ 4475.

[10] X. An, J.C. Yu, Y. Wang, Y. Hu, X. Yu, G. Zhang, J. Mater. Chem. 22 (2012) 85258531.

[11] S.J. Hong, S. Lee, J.S. Jang, J.S. Lee, Energ. Environ. Sci. 4 (2011) 1781-1787.

[12] G. Xi, B. Yue, J. Cao, J. Ye, Chem. - Eur. J. 17 (2011) 5145-5154.
[13] R. Abe, H. Takami, N. Murakami, B. Ohtani, J. Am. Chem. Soc. 130 (2008) $7780-$ 7781.

[14] Y.C. Nah, I. Paramasivam, R. Hahn, N.K. Shrestha, P. Schmuki, Nanotechnology 21 (2010) 105704.

[15] F. Wang, C. Di Valentin, G. Pacchioni, J. Phys. Chem. C 116 (2012) 8901-8909.

[16] Q. Xiang, G.F. Meng, H.B. Zhao, Y. Zhang, H. Li, W.J. Ma, J.Q. Xu, J. Phys. Chem. C 114 (2010) 2049-2055.

[17] Z.G. Zhao, M. Miyauchi, Angew. Chem. Int. Ed. 47 (2008) 7051-7055.

[18] H. Song, Y. Li, Z. Lou, M. Xiao, L. Hu, Z. Ye, L. Zhu, Appl. Catal. B 166 (2015) 112 120.

[19] Y. Liu, J. Li, W. Li, Y. Yang, Y. Li, Q. Chen, J. Phys. Chem. C 119 (2015) 1483414842.

[20] E. Mena, A. Rey, S. Contreras, F.J. Beltran, Catal. Today 252 (2015) 100-106.

[21] W. Zeng, H. Zhang, Z. Wang, Appl. Surf. Sci. 347 (2015) 73-78.

[22] J.Z. Ou, R.A. Rani, S. Balendhran, A.S. Zoolfakar, M.R. Field, S. Zhuiykov, A.P. O'Mullane, K. Kalantar-zadeh, Electrochem. Commun. 27 (2013) 128-132.

[23] D. Tanaka, Y. Oaki, H. Imai, Chem. Commun. 46 (2010) 5286-5288.

[24] G. Xi, Y. Yan, Q. Ma, J. Li, H. Yang, X. Lu, C. Wang, Chem. - Eur. J. 18 (2012) 13949-13953.

[25] F. Amano, D. Li, B. Ohtani, Chem. Commun. 46 (2010) 2769-2771.

[26] D.Y. Zhao, J.L. Feng, Q.S. Huo, N. Melosh, G.H. Fredrickson, B.F. Chmelka, G.D. Stucky, Science 279 (1998) 548-552.

[27] D.Y. Zhao, Q.S. Huo, J.L. Feng, B.F. Chmelka, G.D. Stucky, J. Am. Chem. Soc. 120 (1998) 6024-6036.

[28] S. Perathoner, P. Lanzafame, R. Passalacqua, G. Centi, R. Schlogl, D.S. Su, Micropor. Mesopor. Mater. 90 (2006) 347-361.

[29] W. Wang, M. Song, Micropor. Mesopor. Mater. 96 (2006) 255-261.

[30] M.V.P. Sharma, V.D. Kumari, M. Subrahmanyam, Chemosphere 73 (2008) $1562-1569$.

[31] X.L. Yang, W.L. Dai, R. Gao, K. Fan, J. Catal. 249 (2007) 278-288.

[32] X.L. Yang, W.L. Dai, H. Chen, Y. Cao, H.X. Li, H.Y. He, K.N. Fan, J. Catal. 229 (2005) 259-263.

[33] L. Dimitrov, R. Palcheva, A. Spojakina, K. Jiratova, J. Porous Mater. 18 (2011) 425-434.

[34] E. Kraleva, R. Palcheva, L. Dimitrov, U. Armbruster, A. Brueckner, A. Spojakina, J. Mater. Sci. 46 (2011) 7160-7168.

[35] T.I. Bhuiyan, P. Arudra, M.N. Akhtar, A.M. Aitani, R.H. Abudawoud, M.A. AlYami, S.S. Al-Khattaf, Appl. Catal. A 467 (2013) 224-234.

[36] J.C. Hu, Y.D. Wang, L.F. Chen, R. Richards, W.-M. Yang, Z.C. Liu, W. Xu, Micropor. Mesopor. Mater. 93 (2006) 158-163.

[37] X. Hu, T. Mohamood, W. Ma, C. Chen, J. Zhao, J. Phys. Chem. B 110 (2006) 26012-26018.

[38] L. Ye, J. Liu, Z. Jiang, T. Peng, L. Zan, Appl. Catal. B 142 (2013) 1-7.

[39] J.E. Herrera, J.H. Kwak, J.Z. Hu, Y. Wang, C.H.F. Peden, J. Macht, E. Iglesia, J. Catal. 239 (2006) 200-211.

[40] Y. Cao, J. Wang, M. Kang, Y. Zhu, J. Molecul. Catal. A 381 (2014) 46-53.

[41] Y. Wang, Q.H. Zhang, Y. Ohishi, T. Shishido, K. Takehira, Catal. Lett. 72 (2001) 215-219.

[42] Y. Su, Y.M. Liu, L.C. Wang, M. Chen, Y. Cao, W.L. Dai, H.Y. He, K.N. Fan, Appl. Catal. A 315 (2006) 91-100.

[43] X. Zhang, C. Yuan, M. Li, B. Gao, X. Wang, X. Zheng, J. Non-Cryst. Solids 355 (2009) 2209-2215.

[44] M. Bidaoui, C. Especel, N. Bouchenafa-Saib, D. Duprez, O. Mohammedi, S. Royer, Appl. Catal. A 445 (2012) 14-25.

[45] L. Hu, S. Ji, Z. Jiang, H. Song, P. Wu, Q. Lint, J. Phys. Chem. C 111 (2007) 1517315184.

[46] F. Adam, A. Iqbal, Chem. Eng. J. 171 (2011) 1379-1386.

[47] K. Ketpang, M. Kim, S. Kim, S. Shanmugam, Int. J. Hydrogen Energ. 38 (2013) 9732-9740.

[48] F. Chang, Y. Xie, C. Li, J. Chen, J. Luo, X. Hu, J. Shen, Appl. Surf. Sci. 280 (2013) 967-974.

[49] R. Maheswari, M.P. Pachamuthu, A. Ramanathan, B. Subramaniam, Ind. Eng. Chem. Res. 53 (2014) 18833-18839. 